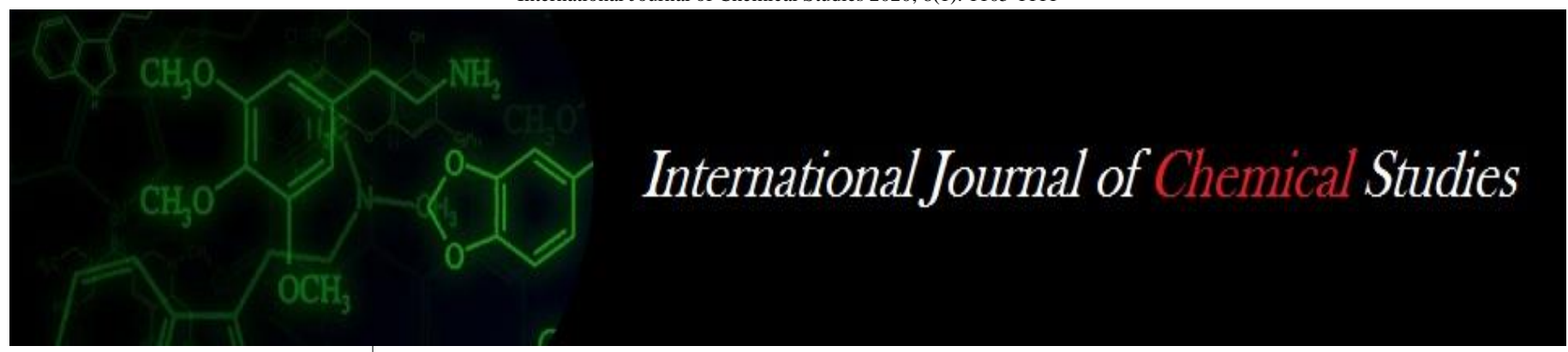

P-ISSN: 2349-8528

E-ISSN: 2321-4902

IJCS 2020; 8(1): 1105-1111

(C) 2020 IJCS

Received: 04-11-2019

Accepted: 06-12-2019

\section{DK Varu}

Professor, Department of

Horticulture, Junagadh

Agricultural Uinversity,

Junagadh, Gujarat, India
Corresponding Author: DK Varu

Professor, Department of Horticulture, Junagadh Agricultural Uinversity, Junagadh, Gujarat, India

\section{Evaluation of various selections on growth, flowering, yield and quality in papaya}

\section{DK Varu}

DOI: https://doi.org/10.22271/chemi.2020.v8.i10.8397

\begin{abstract}
An experiment was conducted to evaluate the different selections along with Pusa Dwarf as a check for growth, flowering, yield and quality traits in papaya. The experiment was conducted at Fruit Research Station, Madhadi bag farm, Department of Horticulture, College of Agriculture, JAU, Junagadh (Gujarat). The study revealed that the maximum number of fruits per plants (36.38) and fruit yield (33.81 $\mathrm{kg} / \mathrm{pl}$. \& 84.52 ton/ha) were noted in Selection-4 (GJP-1). The bearing height is good shine and the check vareity Pusa Dwarf performed with lowest bearing height, but was found at par with Selection-4 (GJP-1). Variation in growth parameters was found significant and lowest plant height and maximum number of leaves/plant were recorded in Pusa Dwarf, while, highest stem girth was noted in Selection-6, but they were observed at par with Selection-4 (GJP-1). Flowering is the main object of plant to target the yield. Significantly the lowest days to flowering was noted in Selection-1 but maturity in Selection-4 (GJP-1). Maximum number of female flower /node was registered in Selection-3, whereas, highest length of pistilate flower, staminate flower and male flower stalk were noted in Selection-6, however, all were found at par with Selection-4(GJP-1). Among the various physical parameters studied, highest fruit length \& weight $(25.02 \mathrm{~cm} \& 1832 \mathrm{~g})$ were noted in Selection-6, whereas, highest fruit girth $(47.3 \mathrm{~cm})$ was noted in Pusa Dwarf but was observed at par with Selection-4 (GJP-1). It was also performed better for highest pulp weight (1327.93 g) and pulp seed ratio (1230.56). Likewise, highest pulp-peel ratio (5.74) was noted in Selection-8, but lowest peel weight $(166.10 \mathrm{~g})$ and seed weight $(63.63 \mathrm{~g})$ were registered in Selection- 2 \& 7. In the present study, Selection-6 \& 4 (GJP-1) established its supremacy in quality parameters viz., TSS, total sugars, reducing sugar, non-reducing sugar over the other varieties. The organolaptic parameters have also great significant to judge the prefarability of the vareity. Highest score of pulp color and taste were noted in Selection- 6 \& 5, respectively, whereas, the highest flavor, texture and over all acceptibility were registerd in Selection-2, however, it was found at par with Selection-4 (GJP-1). Fruit firmness and shelf life of the fruit is also an important feature which enhances the more market price for longer period due to good keeping quality. Highest fruit firmness and shelf life were noted in Selection-7. Papaya Ring Spot Virus (PRSV) is the major devastating disease of papaya. The result was also observed significant and lowest PRSV infestation was noted in Selection-4 (GJP-1).
\end{abstract}

Keywords: Variety, pulp color, fruit length, girth, firmness, shelf life

\section{Introduction}

Papaya is one of the important fruit crops of tropical and sub tropical region of the country. It produces fruits throughout the year. It is easy to cultivate and more remunerative due to higher income per unit area. It ranked second and next to banana. It has a high nutritive and medicinal value especially vitamin A (2020 IU/l00g) (Azad, et al., 2012) ${ }^{[5]}$. It also possesses vitamin B, folate and pantothenic acid besides minerals like potassium and magnesium (Popenoe, 1974) [17]. It is an excellent source of beta carotene which may prevent cancer, diabetes, and heart disease (Aravind et al., 2013) ${ }^{[4]}$ and it is also utilized in the pharmaceutical and cosmetic industries. Papain prepared from dried latex of its immature fruits is used in meat tenderizing, manufacture of chewing gum, cosmetics, degumming, and to give shrink resistance to wool. Besides, it is also used in pharmaceutical industries, textile \& garment, cleaning paper, adhesive manufacture, sewage disposal, etc.

It is quick growing, typically single-stemmed, short-lived, large perennial herb. It is a highly problematic, complicated and interesting fruit crop from botanical, genetically, cytogenetically and horticultural points of view. In India, it is cultivated commercially in 1.33 lakh ha area with 56.39 lakh tonnes production and 42.30 t/ha productivity (Anonymous, 2010). The crop is also highly acclimatized in Gujarat with $5^{\text {th }}$ important fruit crop of Gujarat after mango, 
pomegranate, sapota and acid lime. Gujarat is the second largest in area \& production and fourth in productivity contributing 0.20 lakh ha, 11.85 lakh tonnes and 60.5 t/ha. Respectively (Anonymous, 2010). Hybrids or varieties are the important tools to achieve higher yield and quality. At present, large number of varieties of papaya are cultivated in India. Commercially papaya varieties are grouped in two groups viz., dioecious and gynodioecious. The hybrids/ varieties like Pusa Majesty, Pusa Delicious, Pusa Dwarf, Pusa Nanha, Surya, Coorge Honey Dew, Co-1, Co-2, Co-3, Co-4, Co-5, Co-6, Pink Fleshed sweet, Sunrise Solo, Arka Surya, Arka Prabhat etc. as well as some private sector varieties are commercially cultivated in the country.

Selection is the tools which have a great significant role to crop improvement work which depends on the evaluation of various varieties or selections. Crop improvement work through sib mating \& selection was started earlier and identified promising selection known as Local which was commercially cultivated in the state (Gujarat). There is no public variety in Gujarat. Taiwan varieties like Red Leady, 786, Sweet Charley, etc. are from private sectors under cultivation in Gujarat. Some drawbacks in these varieties with higher price of planting materials were observed from the farmers' feedback. Considering the above facts, the work was started under Crop Improvement Project in papaya at Department of Horticulture, College of Agriculture, JAU, Junagadh to develop the variety.

\section{Materials and Methods}

The experiment was conducted at Department of Horticulture, College of Agriculture, Junagadh Agricultural University, Junagadh. Nine different selections \& cultivar, viz., Selection1 to 8 and Pusha Dwarf (check) were evaluated in Randomized Block Design (RBD) with three replications. The orchard was laid out in square system with $1.8 \times 1.8 \mathrm{~m}$ spacing. Seedlings of different selections and cultivar were raised in nursery. The uniform planting materials i.e. seedlings were used for the present study. All plants were given uniform cultural operation as per the recommended package and practices. The soil of experimental field was sandy loam to alluvial type. The selected plants were marked with metal tag for recording observation. The observations like plant height, bearing height, number of leaves, stem girth, flowering parameters like days to flowering, days to fruit set, days to maturity, number of nodes to first flower, length of inter node, number of female flower bud/node; physical parameters like, fruit length, Fruit girth, Fruit weight, Pulp weight per fruit, Peel weight per fruit, Seed weight per fruit, Pulp-peel ratio \& Pulp-seed ratio; Yield parameters like, number of fruits/plants, fruit yield $(\mathrm{kg} / \mathrm{pl}$. \& ton/ha) and biochemical parameters were recorded. Observations on growth parameters were taken at the beginning, whereas fruit characters where recorded at the time of harvesting. Plant height and stem girth were recorded with measuring tape. The fruits of different selections and cultivar were harvested with twisting the fruit keeping a small intact pedicel with each fruit. The number of fruits per plant were recorded at the time of harvesting from the marked plants. The total fruit yield per plant was obtained through the number of fruits retained by the trees and weighing the fruits by electronic balanced. Fruit size was recorded by measuring length and girth with the help of measuring tap. The rind of freshly harvested fruits was peeled, pulp \& seeds were separated and weighed by using electronic balance. Mean weight was computed and expressed in grams. The biochemical parameters like TSS, sugars, etc. were recorded with prescribed methods. TSS was determined with the help of digital refracto meter. Organoleptic parameters with shelf life were also recorded. The data was statistically analysed by method of analysis of variance using RBD as described by Panse and Sukhatme (1985) ${ }^{[16]}$.

\section{Results and Discussions}

Fruit yield is the most important and polygenic character. Besides, better management of orchard, genetic diversity i.e. variety is another important factor influencing the yield. It is revealed that, the highest number of fruit per plant (36.38) was recorded in Selection-4 (GJP-1) duirng all three years as well as pooled, but was observed at par with Selection- $6 \& 8$ duirng pooled. Similar trend was observed for fruit yield and highest fruit yield $(33.81 \mathrm{~kg} / \mathrm{pl}$. \& $84.52 \mathrm{ton} / \mathrm{ha})$ were noted in Selection-4 (GJP-1) duirng all three years and pooled. However, which was noted at par with Selection-6 \& 8. The variations in yield and yield attributes might be due to different genetic sources with respect to their genetic makeup. It might be also due to various physiological phenomenon, viz. photosynthetic efficiency, rate of translocation of photosynthates from source to sink and photo-respiration that took place in the plant body and different genetic constitution of varieties, which are responsible for expression of genetic characters under a particular set of environment. This is in conformity with the findings of Kumar et al., (2015) [11], Tyagi et al., (2015) ${ }^{[21]}$, Anh et al., (2011) ${ }^{[2]}$ and Meena et al., (2012) ${ }^{[12]}$ in papaya. The bearing height of plant is good shine for the economic value of crop and the check vareity Pusa Dwarf performed with lowest bearing height duirng three years and pooled, but was found at par with Selection-4 (GJP1). Variation in growth parameters like plant height and number of leavse per plant due to different varieties were found significant (Table 2) and lowest plant height (148.16 $\mathrm{cm}$ ) and maximum number of leaves per plant (41.44) were recorded in Pusa Dwarf, respectively. However, it was found at par with Selection-2, Selectio-4 (GJP-1) \& Selectio-5 duiring pooled. Number of nodes per plant and length of internode are also important traits influencing the number of fruits per plant. Similarly, the stem girth affecting the lodging of plant. Minimum number of nodes per plant (18.44), length of internode $(3.63 \mathrm{~cm})$ and highest stem girth $(38.40 \mathrm{~cm})$ were recorded in Selection-4 (GJP-1), Selection-5 and Selection-6, respectively. Several workers hither to have compared

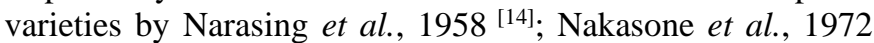
[13]; Selvaraj et al., $1975^{[18]}$ and Ito et al., $1977^{[10]}$ in papaya. Flowering is the main object of plant to target the yield. Significantly the lowest days to flowering (87.03) was noted in Selection-1 but lowest days to maturity (232.33) in Selection-4 (GJP-1). The ancillary observations on flowering were also found significant (Table 5) and maximum number of female flower bud/node (5.84) was registered in Selection3 , but was found at par with Selection-4, $5 \& 8$. Similarly, highest length of pistilate flower bud $(4.48 \mathrm{~cm})$, staminate flower bud $(1.89 \mathrm{~cm})$ and male flower stalk $(33.40 \mathrm{~cm})$ were noted in Selection-6, however, it was found at par with Selection-4 (GJP-1) during all years and pooled.

Length, girth and weight of fruits were the major components of fruit size under the present study (Table 6). The result was also found significant and highest fruit length \& weight $(25.02 \mathrm{~cm} \& 1832 \mathrm{~g}$, respectively) were noted in Selection-6, but was found at par with Selection-4 (GJP-1) and selection-7. Whereas, highest fruit girth $(47.30 \mathrm{~cm})$ was noted in Pusa Dwarf and was observed at par with Selection-2 \& 4 (GJP-1), $5,6 \& 8$. The variation in fruit length, girth and weight might 
be based on the fact that every genotypes has its own nature in development of fruits. It also might be attributed to genetic constitution of the plants. It may also be due to phenotypic and genotypic interactions among the selections. Similar findings were reported by Kumar et al., (2015) ${ }^{[11]}$; Das (2013) [7], Das and Dinesh (2014) ${ }^{[8]}$, Chalak et al., (2016) [6]; Goenaga et al., (2001) [9] and Tyagi et al., (2015) ${ }^{[21]}$ in papaya.

Likewise, highest pulp weight $(1327.93 \mathrm{~g})$ and pulp seed ratio (1230.56) were noted in Selection-4 (GJP-1) and was observed at par with Selection-6,7 \& 8. Lowest peel weight $(166.10 \mathrm{~g})$ and seed weight $(63.63 \mathrm{~g})$ were registered in Selection-2 and Selection-7, respectively. However, highest pulp-peel ratio (5.74) was noted in Selection-8 and which was found at par with Selection-4 (GJP-1), Selection-2, 5 \& 6. Such variation among the selections in pulp, peel \& seed characters may be attributed to genetic makeup of the plants. Seed weight might be due to pollen availability, stigmatic fertility and effective fertilization. Variations in those characters in papaya fruit were also observed in by Nakasone

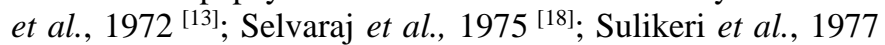

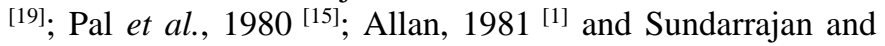
Krishnan, $1984^{[20]}$.

The various bio-chemical components are of utmost important to assess the fruits either for dessert purpose or for processing. Total soluble solids indicates higher sugar content in the fruits and is considered as one of the important criterion for dessert quality whereas caracaxenthin content which causes yellowish orange coloration is important determinant of processing quality. In the present study, Selection-6 and Selection- 4 (GJP-1) established its supremacy in quality parameters viz., total soluble solids $\left(14.52 \& 11.92{ }^{0} \mathrm{~B}\right)$, total sugars $(8.58 \&$
$7.95 \%)$, reducing sugar $(6.03 \& 5.54 \%)$, non-reducing sugar $(2.55 \& 2.41 \%)$, respectively, over the other varieties. It may be due to phenotypic and genetic constitution among the selections which might had necessitated consumption of nutrients and sinking more carbohydrates into the fruits, thus producing larger fruits with more TSS. This is in conformity

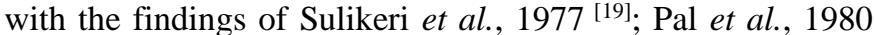
[15]; Allan, $1981^{[1]}$ and Sundarrajan and Krishnan, $1984^{\text {[20]; }}$ Tyagi et al., $2015^{[21]}$. The sugars present in the fruit impart the sweetness while sugars and organic acids present in the fruit influence its taste and flavour. This is in conformity with the findings of Nakasone et al., 1972 [13]; Selvaraj et al., 1975 ${ }^{[18]}$ and Sundarrajan and Krishnan, $1984^{[20]}$. The organolaptic parameters (Table) have also great significant to judge the prefarability of the vareity. Significantly the highest score of pulp color and taste $(7.67 \&$ 7.24) were noted in Selection-6 $\& 5$, respectively. Whereas, the highest flavor, texture and over all acceptibility $(7.23,7.54 \& 7.40)$ were registerd in Selection-2, however, it was found at par with Selection-4 (GJP-1) for all cases. These results are in contrast with Meena et al., (2012) ${ }^{[12]}$.

Fruit firmness and shelf life of the fruit is also an important feature which enhances the more market price for longer period due to good keeping quality. Highest fruit firmness and shelf life $\left(14.17 \mathrm{~kg} / \mathrm{cm}^{2}\right.$ and 4.20 days) were noted in Selection-7 which was observed at par with Selection-3. The shelf life of variety is long mainly due to shininess of fruit.

Papaya Ring Spot Virus (PRSV) is the major devastating disease of papaya. The result was also observed significant and lowest PRSV (15.49\%) was noted in Selection-4 (GJP-1) followed by Selection-1.

Table 1: Evaluation of different selections and cultivar on number of fruits/pls. and fruit yield

\begin{tabular}{|c|c|c|c|c|c|c|c|c|c|c|c|c|}
\hline \multirow{2}{*}{ Selections } & \multicolumn{4}{|c|}{ No. of fruits/pls. } & \multicolumn{4}{|c|}{ Fruit yield (kg/pls.) } & \multicolumn{4}{|c|}{ Fruit yield (t/ha) } \\
\hline & 2013-14 & 2014-15 & 2015-16 & Pooled & 2013-14 & 2014-15 & 2015-16 & Pooled & 2013-14 & 2014-15 & 2015-16 & Pooled \\
\hline Selection-1 & 31.00 & 30.80 & 27.67 & 29.82 & 26.20 & 24.09 & 16.18 & 22.16 & 65.51 & 60.22 & 40.45 & 55.39 \\
\hline Selection-2 & 32.33 & 31.87 & 30.33 & 31.51 & 29.04 & 21.51 & 17.49 & 22.68 & 72.60 & 53.78 & 43.73 & 56.70 \\
\hline Selection-3 & 33.33 & 30.97 & 29.55 & 31.28 & 21.27 & 15.36 & 20.27 & 18.97 & 53.17 & 38.40 & 50.68 & 47.42 \\
\hline Selection-4 & 38.33 & 37.03 & 33.77 & 36.38 & 37.08 & 34.39 & 29.96 & 33.81 & 92.69 & 85.97 & 74.89 & 84.52 \\
\hline Selection-5 & 29.33 & 29.53 & 27.92 & 28.93 & 31.13 & 18.85 & 21.06 & 23.68 & 77.83 & 47.13 & 52.66 & 59.21 \\
\hline Selection-6 & 30.67 & 33.20 & 33.67 & 32.51 & 30.39 & 23.36 & 25.70 & 26.49 & 75.98 & 58.41 & 64.25 & 66.21 \\
\hline Selection-7 & 22.00 & 20.67 & 24.55 & 22.41 & 16.77 & 18.80 & 22.00 & 19.19 & 41.92 & 46.99 & 54.99 & 47.97 \\
\hline Selection- 8 & 37.00 & 32.27 & 28.00 & 32.42 & 31.39 & 26.99 & 23.26 & 27.21 & 78.48 & 67.48 & 58.14 & 68.03 \\
\hline Pusa Dwarf & 35.40 & 33.13 & 26.17 & 31.56 & 27.90 & 20.02 & 15.84 & 21.25 & 69.75 & 50.04 & 39.59 & 53.13 \\
\hline S.Em.+ & 1.678 & 1.385 & 1.279 & 1.605 & 1.643 & 1.185 & 0.785 & 2.498 & 4.106 & 2.963 & 1.963 & 6.246 \\
\hline C. D. at $5 \%$ & 5.03 & 4.15 & 3.84 & 4.81 & 4.92 & 3.55 & 2.35 & 7.49 & 12.31 & 8.88 & 5.88 & 18.73 \\
\hline YxT/S.Em.+ & - & - & - & 1.457 & - & - & - & 1.844 & - & - & - & 3.135 \\
\hline C. D. at $5 \%$ & - & - & - & 4.15 & - & - & - & 5.25 & - & - & - & 8.92 \\
\hline C. V. \% & 9.04 & 7.72 & 7.62 & 8.21 & 10.19 & 9.08 & 6.38 & 9.08 & 10.19 & 9.08 & 6.38 & 9.08 \\
\hline
\end{tabular}

Table 2: Evaluation of different selections and cultivar on growth parameters

\begin{tabular}{|c|c|c|c|c|c|c|c|c|c|c|c|c|}
\hline \multirow{2}{*}{ Selections } & \multicolumn{4}{|c|}{ Plant height (cm) } & \multicolumn{4}{|c|}{ Bearing height $(\mathrm{cm})$} & \multicolumn{4}{|c|}{ No. of leaves per plant } \\
\hline & 2013-14 & 2014-15 & 2015-16 & Pooled & 2013-14 & 2014-15 & 2015-16 & Pooled & 2013-14 & 2014-15 & 2015-16 & Pooled \\
\hline Selection-1 & 184.00 & 166.00 & 181.33 & 177.11 & 68.40 & 64.57 & 75.53 & 69.50 & 28.53 & 26.40 & 36.21 & 30.38 \\
\hline Selection-2 & 181.67 & 151.53 & 163.00 & 165.40 & 77.33 & .27 & 57.60 & 07 & 29.53 & 3.53 & 67 & 91 \\
\hline Selection-3 & 189.00 & 164.67 & 175.67 & 176.44 & 82.67 & 62.97 & 61.67 & 69.10 & 32.00 & 25.67 & 35.00 & 30.89 \\
\hline Selection-4 & 185.00 & 159.17 & 172.67 & 172.28 & 75.67 & 66.03 & 56.80 & 66.17 & 38.60 & 28.53 & 42.02 & 36.38 \\
\hline Selection-5 & 187.33 & 149.40 & 184.33 & 173.69 & 66.67 & 61.30 & 68.13 & 65.37 & 31.20 & 29.87 & 44.33 & 35.13 \\
\hline Selection-6 & 241.33 & 179.60 & 254.00 & 224.98 & 86.93 & 88.00 & 83.47 & 86.13 & 32.40 & 31.60 & 50.42 & 38.14 \\
\hline Selection-7 & 210.33 & 157.37 & 205.67 & 191.12 & 107.60 & 74.03 & 82.53 & 88.06 & 30.60 & 34.53 & 50.30 & 38.48 \\
\hline Selection-8 & 199.00 & 167.97 & 163.33 & 176.77 & 94.13 & 71.13 & 59.57 & 74.94 & 33.27 & 37.60 & 37.33 & 36.07 \\
\hline Pusa Dwarf & 158.00 & 137.13 & 149.33 & 148.16 & 63.87 & 60.47 & 53.93 & 59.42 & 48.33 & 33.20 & 42.78 & 41.44 \\
\hline S.Em.+ & 8.312 & 6.505 & 8.056 & 9.150 & 2.216 & 2.325 & 2.921 & 5.708 & 1.541 & 1.642 & 2.266 & 3.026 \\
\hline C. D. at $5 \%$ & 24.92 & 19.50 & 24.15 & 27.43 & 6.64 & 6.97 & 8.76 & 17.11 & 4.62 & 4.92 & 6.79 & 9.07 \\
\hline YxT/S.Em.+ & - & - & - & 7.617 & - & - & - & 1.844 & - & - & - & 1.844 \\
\hline
\end{tabular}




\begin{tabular}{|c|c|c|c|c|c|c|c|c|c|c|c|c|}
\hline C. D. at $5 \%$ & - & - & - & 21.68 & - & - & - & 5.25 & - & - & - & 5.25 \\
\hline C. V. \% & 7.46 & 7.08 & 7.61 & 9.33 & 4.78 & 5.87 & 7.60 & 7.63 & 7.89 & 9.45 & 9.42 & 12.01 \\
\hline
\end{tabular}

Table 3: Evaluation of different selections and cultivar on growth parameters

\begin{tabular}{|c|c|c|c|c|c|c|c|c|c|c|c|c|}
\hline \multirow{2}{*}{ Selections } & \multicolumn{4}{|c|}{ No. of node per pl. } & \multicolumn{4}{|c|}{ Length of inter node $(\mathrm{cm})$} & \multicolumn{4}{|c|}{ Stem girth $(\mathbf{c m})$} \\
\hline & 2013-14 & 2014-15 & 2015-16 & Pooled & 2013-14 & 2014-15 & 2015-16 & Pooled & 2013-14 & 2014-15 & 2015-16 & Pooled \\
\hline Selection-1 & 32.00 & 27.33 & 28.67 & 29.33 & 5.27 & 4.80 & 5.10 & 5.06 & 31.80 & 25.22 & 24.63 & 27.21 \\
\hline Selection-2 & 21.17 & 18.50 & 19.50 & 19.72 & 4.33 & 4.17 & 4.62 & 4.37 & 35.90 & 27.13 & 25.22 & 29.42 \\
\hline Selection-3 & 22.33 & 19.33 & 20.33 & 20.67 & 5.43 & 4.97 & 4.03 & 4.81 & 38.00 & 26.88 & 31.78 & 32.22 \\
\hline Selection-4 & 19.33 & 17.67 & 18.33 & 18.44 & 4.27 & 4.13 & 5.03 & 4.48 & 36.13 & 27.12 & 31.42 & 31.56 \\
\hline Selection-5 & 20.33 & 21.33 & 23.33 & 21.67 & 3.53 & 3.62 & 3.74 & 3.63 & 36.07 & 27.35 & 31.22 & 31.55 \\
\hline Selection-6 & 27.33 & 23.50 & 24.50 & 25.11 & 7.30 & 7.02 & 7.49 & 7.27 & 45.93 & 32.60 & 36.66 & 38.40 \\
\hline Selection-7 & 24.00 & 21.17 & 22.17 & 22.44 & 6.77 & 6.22 & 7.10 & 6.69 & 40.60 & 35.44 & 37.67 & 37.90 \\
\hline Selection-8 & 22.17 & 19.83 & 20.83 & 20.94 & 4.17 & 4.27 & 4.17 & 4.20 & 37.67 & 29.52 & 28.00 & 31.73 \\
\hline Pusa Dwarf & 22.33 & 21.33 & 23.00 & 22.22 & 3.50 & 3.58 & 4.18 & 3.76 & 40.07 & 26.88 & 30.58 & 32.51 \\
\hline S.Em.+ & 0.931 & 0.978 & 1.152 & 0.592 & 0.167 & 0.177 & 0.101 & 0.215 & 1.611 & 1.538 & 1.373 & 2.313 \\
\hline C. D. at $5 \%$ & 2.79 & 2.93 & 3.45 & 1.68 & 0.50 & 0.53 & 0.30 & 0.64 & 4.83 & 4.61 & 4.12 & 6.94 \\
\hline YxT/S.Em.+ & - & - & - & 1.025 & - & - & - & 0.152 & - & - & - & 1.510 \\
\hline C. D. at $5 \%$ & - & - & - & NS & - & - & - & 0.433 & - & - & - & NS \\
\hline C. V. \% & 6.88 & 8.03 & 8.95 & 10.15 & 5.85 & 6.44 & 3.47 & 6.53 & 7.34 & 9.29 & 7.72 & 8.05 \\
\hline
\end{tabular}

Table 4: Evaluation of different selections and cultivar on days to flowering, fruit maturity \& No. of female flower bud/node

\begin{tabular}{|c|c|c|c|c|c|c|c|c|c|c|c|c|}
\hline \multirow{2}{*}{ Selections } & \multicolumn{4}{|c|}{ Days to flowering } & \multicolumn{4}{c|}{ Days to maturity } & \multicolumn{3}{c|}{ No. of flower bud /node (Female) } \\
\cline { 2 - 16 } & $\mathbf{2 0 1 3 - 1 4}$ & $\mathbf{2 0 1 4 - 1 5}$ & $\mathbf{2 0 1 5 - 1 6}$ & Pooled & $\mathbf{2 0 1 3 - 1 4}$ & $\mathbf{2 0 1 4 - 1 5}$ & $\mathbf{2 0 1 5 - 1 6}$ & Pooled & $\mathbf{2 0 1 3 - 1 4}$ & $\mathbf{2 0 1 4 - 1 5}$ & $\mathbf{2 0 1 5 - 1 6}$ & Pooled \\
\hline Selection-1 & 92.77 & 86.33 & 82.00 & 87.03 & 239.27 & 234.33 & 233.67 & 235.76 & 4.70 & 4.50 & 5.03 & 4.74 \\
\hline Selection-2 & 92.00 & 97.60 & 84.67 & 91.42 & 238.67 & 234.00 & 229.33 & 234.00 & 4.47 & 3.83 & 4.57 & 4.29 \\
\hline Selection-3 & 97.07 & 93.73 & 89.67 & 93.49 & 243.33 & 238.53 & 235.67 & 239.18 & 5.67 & 5.47 & 6.40 & 5.84 \\
\hline Selection-4 & 94.83 & 92.53 & 92.00 & 93.12 & 235.00 & 232.33 & 229.67 & 232.33 & 5.93 & 5.50 & 5.77 & 5.73 \\
\hline Selection-5 & 98.67 & 97.80 & 91.67 & 96.04 & 239.67 & 243.33 & 239.33 & 240.78 & 5.50 & 5.43 & 6.20 & 5.71 \\
\hline Selection-6 & 107.83 & 105.27 & 100.67 & 104.59 & 250.67 & 250.33 & 256.00 & 252.33 & 3.93 & 4.33 & 3.57 & 3.94 \\
\hline Selection-7 & 112.83 & 108.67 & 95.67 & 105.72 & 259.00 & 258.33 & 264.67 & 260.67 & 4.10 & 3.90 & 6.00 & 4.67 \\
\hline Selection-8 & 94.83 & 91.87 & 85.33 & 90.68 & 240.00 & 236.67 & 234.33 & 237.00 & 5.57 & 5.20 & 5.30 & 5.36 \\
\hline Pusa Dwarf & 110.83 & 107.73 & 102.00 & 106.86 & 255.33 & 253.00 & 256.67 & 255.00 & 4.53 & 4.23 & 4.70 & 4.49 \\
\hline S.Em.+ & 2.276 & 2.217 & 1.530 & 2.336 & 4.987 & 5.575 & 3.244 & 2.718 & 0.215 & 0.265 & 0.258 & 0.264 \\
\hline C. D. at 5\% & 6.82 & 6.65 & 4.59 & 7.00 & 14.95 & 16.71 & 9.73 & 7.74 & 0.64 & 0.79 & 0.77 & 0.79 \\
\hline YxT/S.Em.+ & - & - & - & 2.036 & - & - & - & 4.707 & - & - & - & 0.247 \\
\hline C. D. at 5\% & - & - & - & NS & - & - & - & NS & - & - & - & 0.703 \\
\hline C. V. \% & 3.94 & 3.92 & 2.90 & 3.65 & 3.53 & 3.98 & 2.32 & 3.36 & 7.55 & 9.73 & 8.47 & 8.60 \\
\hline
\end{tabular}

Table 5: Evaluation of different selections and cultivar on length of pistilate, staminate and male flower stalk

\begin{tabular}{|c|c|c|c|c|c|c|c|c|c|c|c|c|}
\hline \multirow{2}{*}{ Selections } & \multicolumn{3}{|c|}{ Length of pistilate flower bud (cm) } & \multicolumn{3}{c|}{ Length of staminate flower bud (cm) } & \multicolumn{3}{|c|}{ Length of male flower stalk(cm) } \\
\cline { 2 - 15 } & $\mathbf{2 0 1 3 - 1 4}$ & $\mathbf{2 0 1 4 - 1 5}$ & $\mathbf{2 0 1 5 - 1 6}$ & Pooled & $\mathbf{2 0 1 3 - 1 4}$ & $\mathbf{2 0 1 4 - 1 5}$ & $\mathbf{2 0 1 5 - 1 6}$ & Pooled & $\mathbf{2 0 1 3 - 1 4}$ & $\mathbf{2 0 1 4 - 1 5}$ & $\mathbf{2 0 1 5 - 1 6}$ & Pooled \\
\hline Selection-1 & 3.70 & 3.57 & 3.67 & 3.64 & 1.82 & 1.72 & 1.83 & 1.79 & 20.67 & 17.83 & 19.33 & 19.28 \\
\hline Selection-2 & 3.44 & 3.34 & 3.42 & 3.40 & 1.63 & 1.54 & 1.52 & 1.56 & 25.17 & 23.83 & 24.53 & 24.51 \\
\hline Selection-3 & 3.53 & 3.36 & 3.43 & 3.44 & 1.55 & 1.47 & 1.50 & 1.51 & 26.67 & 23.00 & 25.20 & 24.96 \\
\hline Selection-4 & 4.20 & 4.03 & 4.30 & 4.18 & 2.07 & 1.81 & 1.89 & 1.92 & 32.33 & 31.00 & 31.03 & 31.46 \\
\hline Selection-5 & 3.92 & 3.64 & 4.19 & 3.92 & 1.65 & 1.53 & 1.72 & 1.63 & 29.83 & 30.17 & 33.13 & 31.04 \\
\hline Selection-6 & 4.60 & 4.33 & 4.50 & 4.48 & 2.20 & 1.88 & 1.89 & 1.99 & 34.83 & 32.83 & 32.53 & 33.40 \\
\hline Selection-7 & 4.10 & 4.03 & 4.47 & 4.20 & 1.68 & 1.63 & 1.63 & 1.65 & 26.33 & 24.83 & 26.00 & 25.72 \\
\hline Selection-8 & 4.05 & 3.60 & 3.73 & 3.79 & 1.77 & 1.70 & 1.67 & 1.71 & 23.67 & 24.33 & 20.17 & 22.72 \\
\hline Pusa Dwarf & 4.15 & 3.94 & 3.98 & 4.02 & 1.82 & 1.72 & 1.83 & 1.64 & 26.60 & 23.67 & 25.47 & 25.24 \\
\hline S.Em.+ & 0.206 & 0.179 & 0.151 & 0.104 & 0.043 & 0.044 & 0.033 & 0.044 & 1.038 & 0.707 & 0.723 & 0.783 \\
\hline C. D. at 5\% & 0.62 & 0.54 & 0.45 & 0.30 & 0.13 & 0.13 & 0.10 & 0.13 & 3.11 & 2.12 & 2.17 & 2.35 \\
\hline YxT/S.Em.+ & - & - & - & 0.180 & - & - & - & 0.041 & - & - & - & 0.837 \\
\hline C. D. at 5\% & - & - & - & NS & - & - & - & 0.12 & - & - & - & 2.38 \\
\hline C. V. \% & 9.00 & 8.26 & 6.58 & 8.00 & 4.21 & 4.61 & 3.39 & 4.10 & 6.57 & 4.76 & 4.75 & 5.47 \\
\hline
\end{tabular}

Table 6: Evaluation of different selections and cultivar on fruit length, girth and weight

\begin{tabular}{|c|c|c|c|c|c|c|c|c|c|c|c|c|}
\hline \multirow{2}{*}{ Selections } & \multicolumn{4}{|c|}{ Fruit length (cm) } & \multicolumn{4}{|c|}{ Fruit girth $(\mathbf{c m})$} & \multicolumn{4}{|c|}{ Fruit weight (kg) } \\
\hline & 2013-14 & 2014-15 & 2015-16 & Pooled & 2013-14 & 2014-15 & $2015-16$ & Pooled & 2013-14 & 2014-15 & 2015-16 & Pooled \\
\hline Selection-1 & 26.27 & 19.98 & 23.83 & 23.36 & 43.17 & 32.73 & 39.33 & 38.41 & 1269.07 & 1126.53 & 1454.67 & 1283.42 \\
\hline Selection-2 & 20.60 & 19.53 & 14.70 & 18.28 & 47.20 & 45.71 & 47.93 & 46.95 & 1317.20 & 1060.60 & 1248.33 & 1208.71 \\
\hline Selection-3 & 24.27 & 18.88 & 16.79 & 19.98 & 44.80 & 38.83 & 44.17 & 42.60 & 1174.60 & 797.93 & 1455.00 & 1142.51 \\
\hline Selection-4 & 24.23 & 23.71 & 20.95 & 22.97 & 46.23 & 42.61 & 45.10 & 44.65 & 1810.40 & 1384.03 & 1744.33 & 1646.26 \\
\hline Selection-5 & 21.27 & 17.49 & 16.45 & 18.40 & 47.60 & 45.71 & 44.77 & 46.03 & 1297.70 & 916.53 & 1220.33 & 1144.86 \\
\hline Selection-6 & 28.00 & 24.61 & 22.45 & 25.02 & 45.93 & 45.17 & 46.12 & 45.74 & 1686.40 & 1444.80 & 1832.00 & 1654.40 \\
\hline Selection-7 & 23.07 & 21.22 & 20.60 & 21.63 & 37.13 & 39.20 & 44.57 & 40.30 & 1528.33 & 1325.87 & 1717.67 & 1523.96 \\
\hline
\end{tabular}




\begin{tabular}{|c|c|c|c|c|c|c|c|c|c|c|c|c|}
\hline Selection- 8 & 23.50 & 20.30 & 19.49 & 21.10 & 45.60 & 43.73 & 43.37 & 44.23 & 1620.80 & 1369.13 & 1268.33 & 1419.42 \\
\hline Pusa Dwarf & 18.67 & 20.15 & 18.82 & 19.21 & 44.73 & 46.20 & 50.96 & 47.30 & 1164.00 & 1045.00 & 1536.67 & 1248.56 \\
\hline S.Em.+ & 0.830 & 0.620 & 0.771 & 1.103 & 0.938 & 1.025 & 1.058 & 1.505 & 69.998 & 55.495 & 64.031 & 94.162 \\
\hline C. D. at $5 \%$ & 2.49 & 1.86 & 2.39 & 3.31 & 2.81 & 3.07 & 3.17 & 4.51 & 209.86 & 166.38 & 191.97 & 282.31 \\
\hline YxT/S.Em.+ & - & - & - & 0.745 & - & - & - & 1.008 & - & - & - & 0.063 \\
\hline C. D. at $5 \%$ & - & - & - & 2.12 & - & - & - & 2.87 & - & - & - & 0.18 \\
\hline C. V. \% & 6.16 & 5.20 & 6.90 & 6.12 & 3.63 & 4.20 & 4.06 & 3.97 & 8.48 & 8.26 & 7.41 & 8.06 \\
\hline
\end{tabular}

Table 7: Evaluation of different selections and cultivar on pulp, peel and seed weight

\begin{tabular}{|c|c|c|c|c|c|c|c|c|c|c|c|c|}
\hline \multirow{2}{*}{ Selections } & \multicolumn{4}{|c|}{ Pulp weight (g) } & \multicolumn{4}{|c|}{ Peel weight (g) } & \multicolumn{4}{|c|}{ Seed weight (g) } \\
\hline & 2013-14 & 2014-15 & 2015-16 & Pooled & 2013-14 & 2014-15 & 2015-16 & Pooled & 2013-14 & 2014-15 & 2015-16 & Pooled \\
\hline Selection-1 & 938.83 & 831.07 & 1122.33 & 964.08 & 221.33 & 224.00 & 292.00 & 245.78 & 98.23 & 106.00 & 106.93 & 103.72 \\
\hline Selection-2 & 1056.67 & 799.93 & 951.67 & 936.09 & 148.27 & 189.67 & 160.37 & 166.10 & 100.07 & 88.05 & 96.38 & 94.83 \\
\hline Selection-3 & 917.20 & 552.87 & 1141.93 & 870.67 & 141.87 & 140.93 & 247.60 & 176.80 & 98.40 & 87.85 & 109.18 & 98.47 \\
\hline Selection-4 & 1448.47 & 1131.53 & 1403.80 & 1327.93 & 251.73 & 226.23 & 282.51 & 253.49 & 79.20 & 103.90 & 109.01 & 97.37 \\
\hline Selection-5 & 1104.00 & 666.67 & 1043.73 & 938.13 & 231.67 & 152.43 & 195.92 & 193.34 & 72.67 & 69.10 & 71.05 & 70.94 \\
\hline Selection-6 & 1392.87 & 1077.00 & 1492.74 & 1320.87 & 279.13 & 272.27 & 294.10 & 281.83 & 82.83 & 110.85 & 125.33 & 106.34 \\
\hline Selection-7 & 1160.67 & 979.60 & 1394.25 & 1178.17 & 158.67 & 275.00 & 308.13 & 247.27 & 49.53 & 60.51 & 80.83 & 63.63 \\
\hline Selection- 8 & 1331.73 & 1074.60 & 1053.33 & 1153.22 & 203.20 & 191.83 & 215.25 & 203.43 & 82.27 & 72.44 & 79.04 & 77.92 \\
\hline Pusa Dwarf & 861.00 & 804.33 & 1080.70 & 915.34 & 221.33 & 224.00 & 292.00 & 259.40 & 98.23 & 106.00 & 106.93 & 94.18 \\
\hline S.Em.+ & 39.904 & 33.021 & 46.502 & 79.918 & 8.515 & 10.987 & 14.823 & 24.543 & 1.601 & 2.957 & 3.343 & 6.923 \\
\hline C. D. at $5 \%$ & 119.64 & 99.00 & 139.42 & 239.61 & 25.53 & 32.94 & 44.44 & 73.58 & 4.80 & 8.87 & 10.02 & 20.76 \\
\hline YxT/S.Em.+ & - & - & - & 40.188 & - & - & - & 6.981 & 3.36 & 5.86 & 5.83 & 6.72 \\
\hline C. D. at $5 \%$ & - & - & - & 114.38 & - & - & - & 19.87 & - & - & - & 8.04 \\
\hline C. V. \% & 6.09 & 6.50 & 6.78 & 6.52 & 6.28 & 4.74 & 5.03 & 5.40 & 4.08 & 5.86 & 5.83 & 5.40 \\
\hline
\end{tabular}

Table 8: Evaluation of different selections and cultivar on pulp peel, pulp seed ratio and TSS

\begin{tabular}{|c|c|c|c|c|c|c|c|c|c|c|c|c|}
\hline \multirow{2}{*}{ Selections } & \multicolumn{4}{|c|}{ Pulp peel ratio } & \multicolumn{4}{|c|}{ Pulp seed ratio } & \multicolumn{4}{|c|}{ TSS $\left({ }^{0} B\right)$} \\
\hline & 2013-14 & 2014-15 & $2015-16$ & Pooled & 2013-14 & 2014-15 & 2015-16 & Pooled & 2013-14 & 2014-15 & $2015-16$ & Pooled \\
\hline Selection-1 & 4.25 & 3.71 & 3.85 & 3.94 & 840.61 & 725.07 & 1015.40 & 860.36 & 10.17 & 12.00 & 11.33 & 11.17 \\
\hline Selection-2 & 6.45 & 4.21 & 4.96 & 5.21 & 956.60 & 711.89 & 855.28 & 841.26 & 10.00 & 12.53 & 11.59 & 11.37 \\
\hline Selection-3 & 5.18 & 3.67 & 6.01 & 4.96 & 818.80 & 465.02 & 1032.75 & 772.19 & 10.17 & 10.00 & 14.56 & 11.58 \\
\hline Selection-4 & 5.81 & 5.01 & 5.93 & 5.58 & 1369.27 & 1027.63 & 1294.79 & 1230.56 & 10.33 & 12.27 & 13.15 & 11.92 \\
\hline Selection-5 & 6.97 & 4.37 & 5.74 & 5.69 & 1031.33 & 597.57 & 972.68 & 867.19 & 11.00 & 11.67 & 14.36 & 12.34 \\
\hline Selection-6 & 5.00 & 3.96 & 5.08 & 4.68 & 1310.03 & 966.15 & 1367.41 & 1214.53 & 12.43 & 14.13 & 17.01 & 14.52 \\
\hline Selection-7 & 4.26 & 3.57 & 5.43 & 4.42 & 1111.13 & 919.09 & 1313.42 & 1114.55 & 11.67 & 13.40 & 16.22 & 13.76 \\
\hline Selection- 8 & 6.57 & 5.75 & 4.90 & 5.74 & 1249.47 & 1002.16 & 974.29 & 1075.31 & 10.27 & 11.23 & 14.98 & 12.16 \\
\hline Pusa Dwarf & 3.32 & 4.07 & 3.37 & 3.58 & 782.07 & 716.41 & 965.03 & 821.17 & 10.43 & 11.73 & 12.83 & 11.67 \\
\hline S.Em.+ & 0.265 & 0.226 & 0.237 & 0.402 & 39.834 & 33.543 & 45.371 & 77.066 & 0.298 & 0.247 & 0.348 & 0.882 \\
\hline C. D. at $5 \%$ & 0.79 & 0.68 & 0.71 & 1.21 & 119.43 & 100.57 & 136.03 & 231.05 & 0.89 & 0.74 & 1.04 & 2.64 \\
\hline YxT/S.Em.+ & - & - & - & 0.243 & - & - & - & 39.876 & - & - & - & 0.300 \\
\hline C. D. at $5 \%$ & - & - & - & 0.69 & - & - & - & 113.50 & - & - & - & 0.86 \\
\hline C. V. \% & 8.63 & 9.18 & 8.16 & 8.64 & 6.56 & 7.33 & 7.22 & 7.07 & 4.81 & 3.53 & 4.31 & 4.24 \\
\hline
\end{tabular}

Table 9: Evaluation of different selections and cultivar on pulp peel, pulp seed ratio and TSS

\begin{tabular}{|c|c|c|c|c|c|c|c|c|c|c|c|c|}
\hline \multirow{2}{*}{ Selections } & \multicolumn{4}{|c|}{ Reducing sugar (\%) } & \multicolumn{4}{|c|}{ Non reducing sugar (\%) } & \multicolumn{4}{|c|}{ Total sugar (\%) } \\
\hline & 2013-14 & 2014-15 & $2015-16$ & Pooled & 2013-14 & 2014-15 & $2015-16$ & Pooled & 2013-14 & 2014-15 & $2015-16$ & Pooled \\
\hline Selection-1 & 5.91 & 5.87 & 4.80 & 5.53 & 1.32 & 1.45 & 1.82 & 1.53 & 7.23 & 7.28 & 6.62 & 7.04 \\
\hline Selection-2 & 6.50 & 6.30 & 4.94 & 5.91 & 1.45 & 2.00 & 1.78 & 1.74 & 7.95 & 8.30 & 6.72 & 7.66 \\
\hline Selection-3 & 5.39 & 5.63 & 4.98 & 5.33 & 1.68 & 2.24 & 2.04 & 1.98 & 7.07 & 7.87 & 7.01 & 7.32 \\
\hline Selection-4 & 5.61 & 5.76 & 5.24 & 5.54 & 2.14 & 2.66 & 2.43 & 2.41 & 7.76 & 8.42 & 7.67 & 7.95 \\
\hline Selection-5 & 5.75 & 6.00 & 5.77 & 5.84 & 2.20 & 2.35 & 1.93 & 2.16 & 7.95 & 8.35 & 7.70 & 8.00 \\
\hline Selection-6 & 6.18 & 6.40 & 5.52 & 6.03 & 2.38 & 2.70 & 2.57 & 2.55 & 8.56 & 9.10 & 8.09 & 8.58 \\
\hline Selection-7 & 5.23 & 5.44 & 4.90 & 5.19 & 2.18 & 2.72 & 2.42 & 2.44 & 7.40 & 8.16 & 7.32 & 7.63 \\
\hline Selection- 8 & 5.44 & 5.58 & 4.50 & 5.17 & 1.39 & 1.78 & 2.18 & 1.78 & 6.83 & 7.35 & 6.67 & 6.95 \\
\hline Pusa Dwarf & 5.26 & 5.24 & 5.04 & 5.18 & 1.38 & 2.24 & 1.42 & 1.68 & 6.65 & 7.47 & 6.45 & 6.86 \\
\hline S.Em.+ & 0.219 & 0.138 & 0.100 & 0.212 & 0.068 & 0.098 & 0.085 & 0.159 & 0.167 & 0.180 & 0.149 & 0.214 \\
\hline C. D. at $5 \%$ & 0.66 & 0.41 & 0.30 & 0.64 & 0.20 & 0.29 & 0.25 & 0.48 & 0.50 & 0.54 & 0.45 & 0.64 \\
\hline YxT/S.Em.+ & - & - & - & 0.160 & - & - & - & 0.084 & - & - & - & 0.166 \\
\hline C. D. at $5 \%$ & - & - & - & 0.46 & - & - & - & 0.24 & - & - & - & $\mathrm{NS}$ \\
\hline C. V. \% & 6.64 & 4.13 & 3.40 & 5.02 & 6.53 & 7.60 & 7.13 & 7.20 & 3.85 & 3.88 & 3.61 & 3.80 \\
\hline
\end{tabular}


Table 10: Evaluation of different selections and cultivar on organoleptic score

\begin{tabular}{|c|c|c|c|c|c|c|c|c|c|c|c|c|}
\hline \multirow{2}{*}{ Selections } & \multicolumn{4}{|c|}{ Color of pulp (score) } & \multicolumn{4}{|c|}{ Flavor (score) } & \multicolumn{4}{|c|}{ Texture (score) } \\
\hline & 2013-14 & 2014-15 & 2015-16 & Pooled & 2013-14 & 2014-15 & 2015-16 & Pooled & 2013-14 & 2014-15 & $2015-16$ & Pooled \\
\hline Selection-1 & 5.89 & 6.56 & 6.53 & 6.33 & 5.67 & 6.40 & 6.50 & 6.19 & 5.78 & 6.37 & 6.13 & 6.09 \\
\hline Selection-2 & 7.11 & 7.27 & 7.67 & 7.35 & 7.00 & 7.02 & 7.67 & 7.23 & 7.00 & 7.54 & 8.08 & 7.54 \\
\hline Selection-3 & 6.33 & 6.25 & 6.58 & 6.39 & 6.00 & 5.97 & 6.08 & 6.02 & 6.67 & 6.29 & 6.33 & 6.43 \\
\hline Selection-4 & 7.30 & 7.05 & 7.52 & 7.29 & 6.89 & 7.08 & 6.83 & 6.93 & 6.51 & 7.06 & 7.58 & 7.05 \\
\hline Selection-5 & 6.87 & 6.92 & 7.17 & 6.99 & 7.67 & 7.28 & 6.75 & 7.23 & 8.11 & 7.09 & 6.92 & 7.37 \\
\hline Selection-6 & 7.67 & 7.23 & 8.12 & 7.67 & 6.78 & 7.33 & 6.75 & 6.95 & 7.00 & 6.77 & 7.00 & 6.92 \\
\hline Selection-7 & 7.44 & 6.47 & 7.23 & 7.05 & 6.55 & 6.29 & 6.83 & 6.56 & 6.78 & 6.33 & 6.75 & 6.62 \\
\hline Selection-8 & 7.00 & 7.07 & 8.00 & 7.36 & 6.89 & 7.61 & 6.33 & 6.94 & 7.44 & 7.68 & 6.29 & 7.14 \\
\hline Pusa Dwarf & 6.89 & 6.04 & 5.75 & 6.23 & 6.44 & 5.98 & 6.42 & 6.28 & 6.44 & 6.45 & 6.92 & 6.60 \\
\hline S.Em.+ & 0.185 & 0.170 & 0.202 & 0.222 & 0.185 & 0.186 & 0.189 & 0.225 & 0.144 & 0.176 & 0.182 & 0.272 \\
\hline C. D. at $5 \%$ & 0.56 & 0.51 & 0.60 & 0.66 & 0.55 & 0.56 & 0.57 & 0.67 & 0.43 & 0.53 & 0.55 & 0.82 \\
\hline YxT/S.Em.+ & - & - & - & 0.186 & - & - & - & 0.186 & - & - & - & 0.168 \\
\hline C. D. at $5 \%$ & - & - & - & 0.53 & - & - & - & 0.53 & - & - & - & 0.48 \\
\hline C. V. \% & 4.62 & 4.36 & 4.87 & 4.63 & 4.81 & 4.75 & 4.89 & 4.82 & 3.63 & 4.45 & 4.58 & 4.24 \\
\hline
\end{tabular}

Table 11: Evaluation of different selections and cultivar on organoleptic score and shelf life of fruits

\begin{tabular}{|c|c|c|c|c|c|c|c|c|c|c|c|c|}
\hline \multirow{2}{*}{ Selections } & \multicolumn{4}{|c|}{ Taste (score) } & \multicolumn{3}{c|}{ Overall acceptability (Score) } & \multicolumn{4}{c|}{ Shelf life (Days) } \\
\cline { 2 - 15 } & $\mathbf{2 0 1 3 - 1 4}$ & $\mathbf{2 0 1 4 - 1 5}$ & $\mathbf{2 0 1 5 - 1 6}$ & Pooled & $\mathbf{2 0 1 3 - 1 4}$ & $\mathbf{2 0 1 4 - 1 5}$ & $\mathbf{2 0 1 5 - 1 6}$ & Pooled & $\mathbf{2 0 1 3 - 1 4}$ & $\mathbf{2 0 1 4 - 1 5}$ & $\mathbf{2 0 1 5 - 1 6}$ & Pooled \\
\hline Selection-1 & 5.55 & 6.05 & 6.20 & 5.93 & 6.08 & 6.13 & 6.13 & 6.12 & 3.02 & 2.95 & 3.28 & 3.09 \\
\hline Selection-2 & 7.11 & 7.39 & 7.83 & 7.45 & 7.33 & 7.54 & 7.33 & 7.40 & 3.44 & 3.31 & 3.26 & 3.34 \\
\hline Selection-3 & 5.78 & 5.91 & 6.67 & 6.12 & 6.00 & 6.25 & 6.00 & 6.08 & 3.85 & 3.88 & 3.95 & 3.89 \\
\hline Selection-4 & 7.11 & 7.68 & 7.17 & 7.32 & 6.92 & 7.11 & 7.33 & 7.12 & 3.04 & 2.97 & 2.95 & 2.99 \\
\hline Selection-5 & 7.29 & 7.51 & 6.92 & 7.24 & 7.72 & 7.09 & 6.89 & 7.23 & 3.37 & 3.48 & 3.54 & 3.46 \\
\hline Selection-6 & 7.17 & 6.86 & 7.67 & 7.23 & 6.93 & 6.78 & 7.00 & 6.90 & 3.30 & 3.63 & 3.97 & 3.63 \\
\hline Selection-7 & 6.56 & 5.98 & 7.00 & 6.51 & 6.42 & 6.43 & 6.72 & 6.52 & 4.21 & 4.24 & 4.14 & 4.20 \\
\hline Selection-8 & 7.22 & 7.49 & 6.42 & 7.04 & 7.18 & 7.15 & 6.67 & 7.00 & 3.06 & 3.12 & 3.12 & 3.10 \\
\hline Pusa Dwarf & 5.55 & 6.05 & 6.20 & 6.36 & 6.30 & 6.45 & 6.17 & 6.31 & 3.19 & 3.23 & 3.14 & 3.19 \\
\hline S.Em.+ & 0.149 & 0.170 & 0.190 & 0.238 & 0.158 & 0.153 & 0.181 & 0.131 & 0.167 & 0.231 & 0.117 & 0.103 \\
\hline C. D. at 5\% & 0.45 & 0.51 & 0.57 & 0.72 & 0.47 & 0.46 & 0.54 & 0.39 & 0.50 & 0.69 & 0.35 & 0.29 \\
\hline YxT/S.Em.+ & - & - & - & 0.170 & - & - & - & 0.164 & - & - & - & 0.181 \\
\hline C. D. at 5\% & - & - & - & 0.48 & - & - & - & NS & - & - & - & NS \\
\hline C. V. \% & 3.82 & 4.36 & 4.74 & 4.33 & 4.03 & 3.91 & 4.69 & 4.22 & 8.54 & 11.66 & 5.79 & 9.25 \\
\hline
\end{tabular}

Table 12: Evaluation of different selections and cultivar on organoleptic score

\begin{tabular}{|c|c|c|c|c|c|c|c|}
\hline \multirow{2}{*}{ Selections } & \multicolumn{3}{|c|}{ Fruit firmness (kg/cm2) } & \multicolumn{3}{c|}{ PRSV infection (\%) } \\
\cline { 2 - 7 } & $\mathbf{1}^{\text {st }} \mathbf{d a y s}$ & $\mathbf{2}^{\text {nd }} \mathbf{d a y s}$ & $\mathbf{3}^{\text {rd }} \mathbf{d a y s}$ & $\mathbf{2 0 1 3 - 1 4}$ & $\mathbf{2 0 1 4 - 1 5}$ & $\mathbf{2 0 1 5 - 1 6}$ & Pooled \\
\hline Selection-1 & 13.67 & 6.47 & 4.73 & 8.93 & 12.00 & 27.48 & 16.14 \\
\hline Selection-2 & 15.00 & 11.97 & 7.30 & 10.00 & 12.53 & 46.50 & 23.01 \\
\hline Selection-3 & 15.00 & 15.00 & 13.83 & 6.60 & 9.33 & 60.83 & 25.59 \\
\hline Selection-4 & 15.00 & 9.43 & 5.23 & 10.33 & 12.27 & 23.87 & 15.49 \\
\hline Selection-5 & 15.00 & 14.30 & 8.77 & 9.17 & 11.67 & 48.73 & 23.19 \\
\hline Selection-6 & 15.00 & 13.03 & 8.03 & 10.43 & 14.47 & 27.07 & 17.32 \\
\hline Selection-7 & 15.00 & 14.20 & 14.17 & 10.67 & 13.40 & 33.01 & 19.03 \\
\hline Selection-8 & 15.00 & 6.97 & 3.53 & 7.67 & 11.23 & 39.72 & 19.54 \\
\hline Pusa Dwarf & 15.00 & 10.23 & 6.10 & 10.50 & 12.40 & 25.57 & 16.16 \\
\hline S.Em.+ & 0.444 & 0.393 & 0.275 & 0.435 & 0.558 & 1.230 & 7.135 \\
\hline C. D. at 5\% & NS & 1.18 & 0.82 & 1.30 & 1.67 & 3.69 & 21.39 \\
\hline YxT/S.Em.+ & - & - & - & 8.04 & 7.96 & 5.76 & 9.14 \\
\hline C. D. at 5\% & - & - & - & - & - & - & 1.98 \\
\hline C. . \% $\%$ & 5.18 & 6.03 & 5.98 & 5.84 & 5.78 & 6.15 & 6.87 \\
\hline
\end{tabular}

\section{Conclusion}

On the bases of above study and observations, it is concluded that the Selection-4 is performing better for more number of fruits per plant, higher fruit yiled, medium fruit size with good attractive shape, higher pulp to seed \& peel ratio; quality traits like TSS, reducing \& total sugar with better organoleptic characters. The fruit with yellowish orange colored, soft palatable pulp of Selection-4 (GJP-1) which are the most preferable traits in people resulted in market price.

\section{References}

1. Allan P. Clonal Honey Gold Pawpaws, A horticultural and commercial success. Citrus \& Subtropical Fruit J. 1981; 575:19-53.

2. Anh NT, Trang PN, Hong NTB, Hoan NG. Evaluating agronomic characteristics of twelve local papaya (Carica papaya L.) varieties in Viet Nam. Bull. Inst. Trop. Agr. 2011; 34:15-22.

3. Anonymous. Indian Horticulture Database, 2015. http: //www. Nhb. gov.in. VNR Private Seed Company, 2015. http: //www.VNR.in. 
4. Aravind G, Bhowmik D, Duraival S, Harish G. Traditional and Medicinal uses of Carica papaya. Journal of Medicinal Plant Studies. 2013; 1(1):7-15.

5. Azad MAK, Rabbani MG, Amin L. Plant regeneration and somatic embryogenesis from immature embryos derived through inters pecific hybridization among Carica species. International Journals of Molecular Sciences. 2012; 13:1706517076.

6. Chalak SU, Kamble AB, Bhalekar SG. Evaluation of different papaya cultivars for yield, quality and papaya ring spot disease under pune conditions. J Krishi Vigyan. 2016; 5(1):60-63.

7. Das SC. Studies on papaya cultivation and evaluation of different varieties and Hybrids in Tripura. Asian $\mathbf{J}$ Hort. 2013; 8(2):470474.

8. Das SC, Dinesh MR. Studies on fruit set and germination in some papaya cultivars. Acta Hort. 2014; 1022:87-90.

9. Goenaga R, Irizarry H, Amadora R. Yield and fruit quality of papaya fruits grown at two locations in Puerto and Rico. J Agric. Univ. 2001; 85(3-4):127-134.

10. Ito PJ, Atubra OK, Norman JC. Performance of Hawaiian cultivars of Pawpaw (Carica papaya L.) in Ghana. Acta Hort. 1977; 53:321-324.

11. Kumar M, Prasad Y, Kumar M, Prakash S, Kumar S. Evaluation of genetic variability, genetic advance, heritability and character association for yield and its contributing traits in papaya. Society Plant Research. 2015; 28(2):99-102.

12. Meena BS, Varma LR, Mehta RS. Evaluation of papaya varieties under North Gujarat conditions. Indian J Hort. 2012; 69(1):114-116.

13. Nakasone HY, Crozier JA, Ikehara DK. Evaluation of Waimanalo, a new papaya strain. Technical Bulletin HAES, Hawaii Univ. 1972; 79:12.

14. Narasing RV, Madhava Rao VN, Venkataraman TM. Co1, a new word in papayas, Indian J Hort. 1958; 2(3):35.

15. Pal DK, Subramanyam MD, Divakar NG, Iyer CPA, Selvaraj Y. Studies on the physico-chemical composition of fruits of 12 papaya varieties. J Food Sci. and Tech. 1980; 17(6):254-256.

16. Panse VG, Sukhatme PV. Statistical Methods for Agricultural Workers. $4^{\text {th }}$ ed. ICAR, New Delhi, 1985.

17. Popenoe W. Manual of Tropical and Sub tropical Fruits. Hafner Press, 1974, 225-269.

18. Selvaraj P, Raman KR, Balakrishna R. A study on the performance of Solo Papaya. Prog. Hort. 1975; 7:5-10.

19. Sulikeri GS, Sharanappa K, Rao MM, Bojappa KM. Solo papaya a promising new find to Karnataka. Curr. Res. 1977; 6(9):149-150.

20. Sundarrajan S, Krishnan BM. Improved papaya varieties, Proc. of National Sem. on Papaya and Papain Production, TNAU, 1984, pp. 40-41.

21. Tyagi M, Singh H, Jawandha SK. Performance of papaya cultivars grown under protected conditions. Indian J Hort. 2015; 72(3):334-337. 